\title{
QCD sum rule analysis of the subleading Isgur-Wise form-factor
}

$$
\tau_{1}\left(v \cdot v^{\prime}\right) \text { and } \tau_{2}\left(v \cdot v^{\prime}\right) \text { for } B \rightarrow D_{1} \ell \bar{\nu} \text { and } B \rightarrow D_{2}^{*} \ell \bar{\nu}
$$

\author{
Ming-Qiu Huang, Cheng-Zu Li \\ CCAST (World Laboratory) P.O. Box 8730, Beijing, 100080
}

and Department of Applied Physics, Changsha Institute of Technology, Hunan 410073, China

Yuan-Ben Dai

Institute of Theoretical Physics, Academia Sinica, P.O.Box 2735, Beijing 100080, China

(June 12, 2021)

\begin{abstract}
We present a QCD sum rule calculation of the Isgur-Wise form-factor $\tau_{1}(v$. $\left.v^{\prime}\right)$ and $\tau_{2}\left(v \cdot v^{\prime}\right)$ for the semileptonic decays $B \rightarrow D_{1}(2420) \ell \bar{\nu}$ and $B \rightarrow$ $D_{2}^{*}(2460) \ell \bar{\nu}$ in the framework of heavy quark effective theory. These two universal functions, associated with the matching of the weak currents in QCD onto those in the effective theory, appear at the order $1 / m_{Q}$ in the heavy quark expansion of meson weak decay form factors.

PACS number(s): 14.40.-n, 12.39.Hg, 11.55.Hx, 12.38.Lg
\end{abstract}

Typeset using REVTEX 


\section{INTRODUCTION}

The heavy quark effective theory (HQET) [1,2] has important consequences on the spectroscopy and the decay matrix elements of the hadrons containing a single heavy quark $Q$. In the infinite mass limit, the spin and parity of the heavy quark and that of the light degrees of freedom are separately conserved. This allows that the hadronic states can be classified in degenerate doublets by the total angular momentum $j$ and the angular momentum of the light degrees of freedom $j_{\ell}$. In the case of $\bar{q} Q$ mesons, coupling $j_{\ell}$ with the spin of heavy quark $s_{Q}=1 / 2$ yields a doublet with total spin $j=j_{\ell} \pm 1 / 2$. The ground state mesons with $j_{\ell}^{P}=\frac{1}{2}^{-}$are the doublet $\left(D, D^{*}\right)$ for $Q=c$ and $\left(B, B^{*}\right)$ for $Q=b$. The excited heavy mesons with $j_{\ell}^{P}=1^{+} / 2$ and $3^{+} / 2$ can be classified in two doublets of spin symmetry $\left(0^{+}, 1^{+}\right)$and $\left(1^{+}, 2^{+}\right)$, respectively. The charmed mesons with $j_{\ell}^{P}=3^{+} / 2$ have been experimentally observed. For $q=u, d$, these mesons are denoted as the $D_{1}(2420)$ and the $D_{2}^{*}(2460)$ mesons. While the $j_{\ell}^{P}=1^{+} / 2$ doublet $\left(D_{0}^{\prime}, D_{1}^{\prime}\right)$ has not been observed yet.

One of the most important applications of the heavy quark symmetries has been the study of semileptonic transitions between two heavy hadrons. The hadronic matrix elements of weak currents between members of the doublets identified by $j_{\ell}$ and $j_{\ell^{\prime}}$ can be expressed in terms of universal form factors which are funtions of the dot-product, $y=v \cdot v^{\prime}$, of the initial and final hadron four-velocities. A well-known result is that the semileptonic decays $B \rightarrow D l \bar{\nu}$ and $B \rightarrow D^{*} l \bar{\nu}$, in the $m_{Q} \rightarrow \infty$ limit,can be described in terms of a single universal function, the Isgur-Wise function $\xi(y)$. In the case of transitions between members belonging to different heavy quark multiplets, additional form factors needs to be introduced. For example, for the $B$ semileptonic decay into excited charmed mesons $\left(D_{0}^{\prime}\right.$, $\left.D_{1}^{\prime}\right)$ and $\left(D_{1}, D_{2}^{*}\right)$ the weak matrix elements can be expressed in terms of two independent functions, $\tau(y)$ and $\zeta(y)$ [3], 4 , respectively, in the limit $m_{Q} \rightarrow \infty$.

There are $\Lambda_{\mathrm{QCD}} / m_{Q}$ corrections to the weak matrix elements parametrized by form factors at the $m_{Q} \rightarrow \infty$ limit. The $\Lambda_{\mathrm{QCD}} / m_{Q}$ corrections to the leading term can be analyzed in a systematical way in HQET. The matrix elements receive contributions from higher-dimensional operators in the effective currents and in the effective Lagrangian. They result in a set of new Isgur-Wise functions.

The universal functions must be estimated in some nonperturbative approaches. A viable approache is the QCD sum rules formulated in the framework of HQET. This method allows to relate hadronic observables to QCD parameters via the operator product expansion (OPE) of the correlator. A fruitful application of QCD sum rules has been the determination of the Isgur-Wise functions parameterizing the $B \rightarrow D^{(*)}$ semileptonic transitions up to the $\Lambda_{\mathrm{QCD}} / m_{Q}$ corrections [5].

The semileptonic $B$ decays into excited charmed mesons have attracted attention in recent years. From the phenomenological point of view, the $B \rightarrow D^{* *}$ transitions are inter- 
esting (here $D^{* *}$ denotes the generic $L=1$ charmed state), since in principle these decay modes may account for a sizeable fraction of the inclusive semileptonic $B$-decay rate. At leading order in the $1 / m_{Q}$ expansion, the two independent universal form factors, $\tau(y)$ and $\zeta(y)$, that parametrize the transitions $B \rightarrow\left(D_{1}, D_{2}^{*}\right) \ell \bar{\nu}$ and $B \rightarrow\left(D_{0}^{\prime}, D_{1}^{\prime}\right) \ell \bar{\nu}$, respectively, have been calculated with QCD sum rules [8,9]. Moreover, perturbative corrections to $\mathcal{O}\left(\alpha_{s}\right)$ have been included in the QCD sum rule for $\zeta(y)$ in [10]. The other approaches include different quark models [11-17] and relativistic Bethe-Salpeter equations [18]. A

model independent analysis has been carried out in 4 for the $\Lambda_{\mathrm{QCD}} / m_{Q}$ corrections. At the order $1 / m_{Q}$, the corrections for matrix elements of $B \rightarrow D^{* *}$ include contributions from higher-dimensional operators in the effective currents and in the effective Lagrangian. For the semileptonic transitions $B \rightarrow D_{1} \ell \bar{\nu}$ and $B \rightarrow D_{2}^{*} \ell \bar{\nu}$, the former give rise to two independent universal functions, denoted by $\tau_{1}(y)$ and $\tau_{2}(y)$ [4]. In the present work we shall focus on the first type of corrections and investigate these two form factors, $\tau_{1,2}(y)$, with QCD sum rules in HQET.

The remainder of this paper is organized as follows. In Sec. II we review the formulae for the matrix elements of the weak currents including the structure of the $\Lambda_{\mathrm{QCD}} / m_{Q}$ corrections in the effective theory. The QCD sum rule analysis for the subleading IsgurWise functions related to the corrections from matching weak currents in QCD onto those in HQET currents is presented in Sec. [II]. Sec. $\mathbb{\text { IV }}$ is devoted to numerical results and our conclusions.

\section{THE HEAVY-QUARK EXPANSION AND THE SUBLEADING ISGUR-WISE FORM FACTORS}

The theoretical description of semileptonic decays involves the matrix elements of vector and axial vector currents $\left(V^{\mu}=\bar{c} \gamma^{\mu} b\right.$ and $\left.A^{\mu}=\bar{c} \gamma^{\mu} \gamma_{5} b\right)$ between $B$ mesons and excited $D$ mesons. For the processes $B \rightarrow D_{1} \ell \bar{\nu}$ and $B \rightarrow D_{2}^{*} \ell \bar{\nu}$, these matrix elements can be parameterized as

$$
\begin{aligned}
& \left\langle D_{1}\left(v^{\prime}, \epsilon\right)\left|V^{\mu}\right| B(v)\right\rangle=f_{V_{1}} \epsilon^{* \mu}+\left(f_{V_{2}} v^{\mu}+f_{V_{3}} v^{\prime \mu}\right) \epsilon^{*} \cdot v \\
& \left\langle D_{1}\left(v^{\prime}, \epsilon\right)\left|A^{\mu}\right| B(v)\right\rangle=i f_{A} \varepsilon^{\mu \alpha \beta \gamma} \epsilon_{\alpha}^{*} v_{\beta} v_{\gamma}^{\prime} \\
& \left\langle D_{2}^{*}\left(v^{\prime}, \epsilon\right)\left|A^{\mu}\right| B(v)\right\rangle=k_{A_{1}} \epsilon^{* \mu \alpha} v_{\alpha}+\left(k_{A_{2}} v^{\mu}+k_{A_{3}} v^{\prime \mu}\right) \epsilon_{\alpha \beta}^{*} v^{\alpha} v^{\beta} \\
& \left\langle D_{2}^{*}\left(v^{\prime}, \epsilon\right)\left|V^{\mu}\right| B(v)\right\rangle=i k_{V} \varepsilon^{\mu \alpha \beta \gamma} \epsilon_{\alpha \sigma}^{*} v^{\sigma} v_{\beta} v_{\gamma}^{\prime} .
\end{aligned}
$$

The form factors $f_{i}$ and $k_{i}$ are functions of $y=v \cdot v^{\prime}$, which can be expressed by a set of Isgur-Wise functions at each order in $\Lambda_{\mathrm{QCD}} / m_{c, b}$. In the infinite mass limit a convenient way to evaluate hadronic matrix elements is by using the covariant trace formalism developed in Ref. [19]. The heavy mesons can be described by spin wave-functions with well-defined 
transformation properties under the Lorentz group and heavy-quark symmetry. The spin doublet parametrized by $j_{\ell}^{P}$ can be represented by a $4 \times 4$ Dirac-type matrix. For $j_{\ell}^{P}=1^{-} / 2$ and $3^{+} / 2$, the matrix representation are

$$
\begin{aligned}
& H_{v}=\frac{1+\psi}{2}\left[P_{v}^{* \mu} \gamma_{\mu}-P_{v} \gamma_{5}\right] \\
& F_{v}^{\mu}=\frac{1+\psi}{2}\left\{P_{v}^{* \mu \nu} \gamma_{\nu}-\sqrt{\frac{3}{2}} P_{v}^{\nu} \gamma_{5}\left[g_{\nu}^{\mu}-\frac{1}{3} \gamma_{\nu}\left(\gamma^{\mu}-v^{\mu}\right)\right]\right\}
\end{aligned}
$$

where $P_{v}, P_{v}^{* \mu}$ and $P_{v}^{\nu}, P_{v}^{* \mu \nu}$ are annihilation operators for members of the $j_{\ell}^{P}=1^{-} / 2$ and $3^{+} / 2$ doublets with four-velocity $v$ in HQET. The matrices $H$ and $F$ satisfy $\psi H_{v}=H_{v}=$ $-H_{v} \psi, \quad \psi F_{v}^{\mu}=F_{v}^{\mu}=-F_{v}^{\mu} \psi, \quad F_{v}^{\mu} \gamma_{\mu}=0$, and $v_{\mu} F_{v}^{\mu}=0$.

Up to the order $\mathcal{O}\left(1 / m_{c, b}\right)$ the current $\bar{c} \Gamma b$ is represented in HQET by

$$
\bar{c} \Gamma b=\bar{h}_{v^{\prime}}^{(c)}\left(\Gamma-\frac{i}{2 m_{c}} \overleftarrow{\not D} \Gamma+\frac{i}{2 m_{b}} \Gamma \overrightarrow{\not D}\right) h_{v}^{(b)}
$$

where $h_{v}^{(Q)}$ is the heavy quark field in the effective theory. Hadronic matrix elements of the leading order current between the states annihilated by the fields in $H_{v}$ and $F_{v^{\prime}}^{\sigma}$ are written as

$$
\bar{h}_{v^{\prime}}^{(c)} \Gamma h_{v}^{(b)}=\tau \operatorname{Tr}\left\{v_{\sigma} \bar{F}_{v^{\prime}}^{\sigma} \Gamma H_{v}\right\}
$$

Here $\tau$ is a universal Isgur-Wise function of $y$.

At order $\Lambda_{\mathrm{QCD}} / m_{c, b}$ there are corrections originating from the matching of the $b \rightarrow c$ flavor changing current onto those in the effective theory. For matrix elements between the states annihilated by the fields in $F_{v^{\prime}}^{\sigma}$ and $H_{v}$, the new operators in Eq. (3) at the order $\Lambda_{\mathrm{QCD}} / m_{c, b}$ can be written as

$$
\begin{aligned}
& \bar{h}_{v^{\prime}}^{(c)} i \overleftarrow{D}_{\lambda} \Gamma h_{v}^{(b)}=\operatorname{Tr}\left\{\mathcal{S}_{\sigma \lambda}^{(c)} \bar{F}_{v^{\prime}}^{\sigma} \Gamma H_{v}\right\} \\
& \bar{h}_{v^{\prime}}^{(c)} \Gamma i \vec{D}_{\lambda} h_{v}^{(b)}=\operatorname{Tr}\left\{\mathcal{S}_{\sigma \lambda}^{(b)} \bar{F}_{v^{\prime}}^{\sigma} \Gamma H_{v}\right\}
\end{aligned}
$$

The most general decomposition of the form factor is

$$
\mathcal{S}_{\sigma \lambda}^{(Q)}=v_{\sigma}\left[\tau_{1}^{(Q)} v_{\lambda}+\tau_{2}^{(Q)} v_{\lambda}^{\prime}+\tau_{3}^{(Q)} \gamma_{\lambda}\right]+\tau_{4}^{(Q)} g_{\sigma \lambda}
$$

The functions $\tau_{i}$ depend on $y$ and have mass dimension one. Using the equation of motion for the heavy quark, $i v \cdot D h_{v}^{(Q)}=0$, and translation invariance, $i \partial_{\nu}\left(\bar{h}_{v^{\prime}}^{(c)} \Gamma h_{v}^{(b)}\right)=\left(\bar{\Lambda} v_{\nu}-\right.$ $\left.\bar{\Lambda}^{\prime} v_{\nu}^{\prime}\right) \bar{h}_{v^{\prime}}^{(c)} \Gamma h_{v}^{(b)}$, one can obtain the constraints [4]

$$
\begin{aligned}
& y \tau_{1}^{(c)}+\tau_{2}^{(c)}-\tau_{3}^{(c)}=0, \\
& \tau_{1}^{(b)}+y \tau_{2}^{(b)}-\tau_{3}^{(b)}+\tau_{4}^{(b)}=0 .
\end{aligned}
$$


and relations between $\tau_{j}^{(b)}$ and $\tau_{j}^{(c)}$

$$
\begin{array}{ll}
\tau_{1}^{(c)}+\tau_{1}^{(b)}=\bar{\Lambda} \tau, & \tau_{2}^{(c)}+\tau_{2}^{(b)}=-\bar{\Lambda}^{\prime} \tau, \\
\tau_{3}^{(c)}+\tau_{3}^{(b)}=0, & \tau_{4}^{(c)}+\tau_{4}^{(b)}=0,
\end{array}
$$

where $m_{Q}+\bar{\Lambda}$ and $m_{Q}+\bar{\Lambda}^{\prime}$ are masses of doublets $j_{\ell}^{P}=1^{-} / 2$ and $3^{+} / 2$ in the leading order. Furthermore, combining Eqs. (7) with (8) yields

$$
\begin{aligned}
& \tau_{3}^{(c)}=y \tau_{1}^{(c)}+\tau_{2}^{(c)}, \\
& \tau_{4}^{(c)}=(y-1)\left(\tau_{1}^{(c)}-\tau_{2}^{(c)}\right)-\left(y \bar{\Lambda}^{\prime}-\bar{\Lambda}\right) \tau .
\end{aligned}
$$

These relations show that all corrections to the form factors coming from the matching of the weak currents in QCD onto those in the effective theory are expressible in terms of $\bar{\Lambda} \tau$ and $\bar{\Lambda}^{\prime} \tau$ and two of the coefficient functions, say, $\tau_{1}^{(c)}$ and $\tau_{2}^{(c)}$.

Applying the above relations to the $1 / m_{c}$ correction to the vector and axial-vector currents . and dropping the superscript $c$ on $\tau_{1,2}^{(c)}$, the corresponding matrix elements can be written as

$$
\begin{aligned}
& \left\langle D_{1}\left(v^{\prime}, \epsilon\right)\left|\bar{h}_{v^{\prime}}^{(c)} i \overleftarrow{\not D} \gamma^{\mu} h_{v}^{(b)}\right| B(v)\right\rangle=\frac{1}{\sqrt{6}}\left\{\left[4(y+1)\left(\bar{\Lambda}^{\prime} y-\bar{\Lambda}\right) \tau-3\left(y^{2}-1\right)\left(\tau_{1}-\tau_{2}\right)\right] \epsilon^{* \mu}\right. \\
& +\left\{\left[(4 y-1) \tau_{1}+5 \tau_{2}\right] v^{\mu}-\left[4\left(\bar{\Lambda}^{\prime} y-\bar{\Lambda}\right) \tau+(y+2) \tau_{1}\right.\right. \\
& \left.\left.\left.+(3 y+2) \tau_{2}\right] v^{\prime \mu}\right\} \epsilon^{*} \cdot v\right\} \text {, } \\
& \left\langle D_{1}\left(v^{\prime}, \epsilon\right)\left|\bar{h}_{v^{\prime}}^{(c)} i \overleftarrow{D D} \gamma^{\mu} \gamma_{5} h_{v}^{(b)}\right| B(v)\right\rangle=\frac{i}{\sqrt{6}}\left[4\left(\bar{\Lambda}^{\prime} y-\bar{\Lambda}\right) \tau-3(y-1)\left(\tau_{1}-\tau_{2}\right)\right] \varepsilon^{\mu \alpha \beta \gamma} \epsilon_{\alpha}^{*} v_{\beta} v_{\gamma}^{\prime} \\
& \left\langle D_{2}^{*}\left(v^{\prime}, \epsilon\right)\left|\bar{h}_{v^{\prime}}^{(c)} i \overleftarrow{D D} \gamma^{\mu} h_{v}^{(b)}\right| B(v)\right\rangle=i\left(\tau_{1}-\tau_{2}\right) \varepsilon^{\mu \alpha \beta \gamma} \epsilon_{\alpha \sigma}^{*} v^{\sigma} v_{\beta} v_{\gamma}^{\prime} \\
& \left.\left\langle D_{2}^{*}\left(v^{\prime}, \epsilon\right)\left|\bar{h}_{v^{\prime}}^{(c)} i \overleftarrow{\not D} \gamma^{\mu} \gamma_{5} h_{v}^{(b)}\right| B(v)\right\rangle=(y-1)\left(\tau_{1}-\tau_{2}\right) \epsilon^{* \mu \alpha} v_{\alpha}+\left[2 \tau_{1} v^{\mu}+\left(\tau_{1}+\tau_{2}\right) v^{\prime \mu}\right)\right] \\
& \times \epsilon_{\alpha \beta}^{*} v^{\alpha} v^{\beta} \text {. }
\end{aligned}
$$

The analogous formulae for the matrix elements of the $1 / m_{b}$ correction to the currents can be written down in a similar way.

There are also order $\Lambda_{\mathrm{QCD}} / m_{c, b}$ corrections originating from terms in the HQET Lagrangian of this order. They can be parametrized by additional functions of $y$ [⿶]. From now on we shall confine our attention to the determination of subleading Isgur-Wise functions, $\tau_{1}(y)$ and $\tau_{2}(y)$, associated with the matching of the vector and axial vector currents in full QCD onto those in the effective theory.

\section{SUM RULES FOR $\tau_{1}$ AND $\tau_{2}$}

A basic element in the application of QCD sum rules to problems involving excited heavy mesons is to choose a set of appropriate interpolating currents in terms of quark 
fields each of which creates (annihilate) an excited state of the heavy meson with definite quantum numbers $j, P, j_{\ell}$. The proper interpolating current $J_{j, P, j_{\ell}}^{\alpha_{1} \cdots \alpha_{j}}$ for the state with arbitrary quantum number $j, P, j_{\ell}$ in HQET was given in 20]. These currents have nice properties. They were proved to satisfy the following conditions

$$
\begin{aligned}
\left\langle 0\left|J_{j, P, j_{\ell}}^{\alpha_{1} \cdots \alpha_{j}}(0)\right| j^{\prime}, P^{\prime}, j_{\ell}^{\prime}\right\rangle= & i f_{P j_{l}} \delta_{j j^{\prime}} \delta_{P P^{\prime}} \delta_{j \ell} j_{\ell}^{\prime} \eta^{\alpha_{1} \cdots \alpha_{j}} \\
i\left\langle 0\left|T\left(J_{j, P, j_{\ell}}^{\alpha_{1} \cdots \alpha_{j}}(x) J_{j^{\prime}, P^{\prime}, j_{\ell}^{\prime}}^{\beta_{1} \cdots \beta_{j^{\prime}}}(0)\right)\right| 0\right\rangle= & \delta_{j j^{\prime}} \delta_{P P^{\prime}} \delta_{j_{\ell} j_{\ell}^{\prime}}(-1)^{j} \mathcal{S} g_{t}^{\alpha_{1} \beta_{1}} \cdots g_{t}^{\alpha_{j} \beta_{j}} \\
& \times \int d t \delta(x-v t) \Pi_{P, j_{\ell}}(x)
\end{aligned}
$$

in the $m_{Q} \rightarrow \infty$ limit. Where $\eta^{\alpha_{1} \cdots \alpha_{j}}$ is the polarization tensor for the spin $j$ state, $v$ is the velocity of the heavy quark, $g_{t}^{\alpha \beta}=g^{\alpha \beta}-v^{\alpha} v^{\beta}$ is the transverse metric tensor, $\mathcal{S}$ denotes symmetrizing the indices and subtracting the trace terms separately in the sets $\left(\alpha_{1} \cdots \alpha_{j}\right)$ and $\left(\beta_{1} \cdots \beta_{j}\right), f_{P, j_{\ell}}$ and $\Pi_{P, j_{\ell}}$ are a constant and a function of $x$ respectively which depend only on $P$ and $j_{\ell}$. Because of equations (11) and (12), the sum rules in HQET for decay amplitudes derived from a correlator containing such currents receive contribution only from one of the two states with the same spin-parity $(j, P)$ but different $j_{\ell}$ in the $m_{Q} \rightarrow \infty$. Starting from the calculations in the leading order, the decay amplitudes for finite $m_{Q}$ can be calculated unambiguously order by order in the $1 / m_{Q}$ expansion in HQET.

Following [20] the local interpolating current for creating $0^{-}$pseudoscalar $B$ meson is taken as

$$
J_{0,-, 1 / 2}^{\dagger \alpha}=\sqrt{\frac{1}{2}} \bar{h}_{v} \gamma_{5} q,
$$

and the local interpolating currents for creating $1^{+}$and $2^{+}\left(D_{1}, D_{2}^{*}\right)$ mesons in the doublet $\left(D_{1}, D_{2}^{*}\right)$ are taken as

$$
\begin{aligned}
J_{1,+, 3 / 2}^{\dagger \alpha} & =\sqrt{\frac{3}{4}} \bar{h}_{v} \gamma^{5}(-i)\left(\mathcal{D}_{t}^{\alpha}-\frac{1}{3} \gamma_{t}^{\alpha} \mathcal{D}_{t}\right) q \\
J_{2,+, 3 / 2}^{\dagger \alpha_{1}, \alpha_{2}} & =\sqrt{\frac{1}{2}} \bar{h}_{v} \frac{(-i)}{2}\left(\gamma_{t}^{\alpha_{1}} \mathcal{D}_{t}^{\alpha_{2}}+\gamma_{t}^{\alpha_{2}} \mathcal{D}_{t}^{\alpha_{1}}-\frac{2}{3} g_{t}^{\alpha_{1} \alpha_{2}} \mathcal{D}_{t}\right) q
\end{aligned}
$$

where $\mathcal{D}$ is the covariant derivative and $\gamma_{t}^{\mu}=\gamma^{\mu}-\psi v^{\mu}$. Note that, without the last term in the bracket in (14) the current would couple also to the $1^{+}$state in the doublet $\left(0^{+}, 1^{+}\right)$ even in the limit of infinite $m_{Q}$.

The QCD sum rule analysis for the subleading form factors, $\tau_{1}(y)$ and $\tau_{2}(y)$, proceeds along the same lines as that for the leading order Isgur-Wise function $\tau(y)$. Here we shall briefly outline the the procedure for determining the Isgur-Wise function $\tau(y)$ and refer for details to Ref. [9]. The idea is to study the analytic properties of the three-point correlators 
$i^{2} \int d^{4} x d^{4} z e^{i\left(k^{\prime} \cdot x-k \cdot z\right)}\left\langle 0\left|T\left(J_{1,+, 3 / 2}^{\nu}(x) \mathcal{J}_{V, A}^{\mu\left(v, v^{\prime}\right)}(0) J_{0,-, 1 / 2}^{\dagger}(z)\right)\right| 0\right\rangle=\Xi\left(\omega, \omega^{\prime}, y\right) \mathcal{L}_{V, A}^{\mu \nu}$,
$i^{2} \int d^{4} x d^{4} z e^{i\left(k^{\prime} \cdot x-k \cdot z\right)}\left\langle 0\left|T\left(J_{2,+, 3 / 2}^{\alpha \beta}(x) \mathcal{J}_{V, A}^{\mu\left(v, v^{\prime}\right)}(0) J_{0,-, 1 / 2}^{\dagger}(z)\right)\right| 0\right\rangle=\Xi\left(\omega, \omega^{\prime}, y\right) \mathcal{L}_{V, A}^{\mu \alpha \beta}$,

where $\mathcal{J}_{V}^{\mu\left(v, v^{\prime}\right)}=\bar{h}\left(v^{\prime}\right) \gamma^{\mu} h(v)$ and $\mathcal{J}_{A}^{\mu\left(v, v^{\prime}\right)}=\bar{h}\left(v^{\prime}\right) \gamma^{\mu} \gamma_{5} h(v)$ are leading order vector and axial vector currents, respectively. The variables $k, k^{\prime}$ denote residual "off-shell" momenta which are related to the momenta $p$ of the heavy quark in the initial state and $p^{\prime}$ in the final state by $k=p-m_{Q} v, k^{\prime}=p^{\prime}-m_{Q^{\prime}} v^{\prime}$, respectively. For heavy quarks in bound states they are typically of order $\Lambda_{Q C D}$ and remain finite in the heavy quark limit. $\mathcal{L}_{V, A}$ are Lorentz structures associated with the vector and axial vector currents.

The coefficient $\Xi\left(\omega, \omega^{\prime}, y\right)$ in (16) is an analytic scalar function in the "off-shell energies" $\omega=2 v \cdot k$ and $\omega^{\prime}=2 v^{\prime} \cdot k^{\prime}$ with discontinuities for positive values of these variables. It furthermore depends on the velocity transfer $y=v \cdot v^{\prime}$, which is fixed at its physical region for the process under consideration. By saturating the double dispersion integrals for the correlators in (16) with physical intermediate states in HQET, one finds the hadronic representation of the correlator as following

$$
\Xi_{\text {hadro }}\left(\omega, \omega^{\prime}, y\right)=\frac{f_{-, \frac{1}{2}} f_{+, 3 / 2} \tau(y)}{\left(2 \bar{\Lambda}_{-, 1 / 2}-\omega-i \epsilon\right)\left(2 \bar{\Lambda}_{+, 3 / 2}-\omega^{\prime}-i \epsilon\right)}+\text { higher resonances },
$$

where $f_{P, j_{\ell}}$ are constants defined in (11), $\bar{\Lambda}_{P, j_{\ell}}=m_{P, j_{\ell}}-m_{Q}$. As the result of equation (11), only one state with $j^{P}=1^{+}$or $j^{P}=2^{+}$contributes to (17), the other resonance with the same quantum number $j^{P}$ and different $j_{l}$ does not contribute.

Following the usual procedure of QCD sum rules and making double Borel transformations in the variables $\omega$ and $\omega^{\prime}$, one obtains the sum rule for $\tau$ as follows

$$
\begin{aligned}
\tau(y) f_{-, 1 / 2} & f_{+, 3 / 2} e^{-2\left(\bar{\Lambda}_{-, 1 / 2} / T_{1}+\bar{\Lambda}_{+, 3 / 2} / T_{2}\right)}=\int_{D} d \nu d \nu^{\prime} \rho^{\text {pert }}\left(\nu, \nu^{\prime}, y\right) e^{-\nu / T_{1}-\nu^{\prime} / T_{2}} \\
- & \frac{m_{0}^{2}}{6} \frac{\langle\bar{q} q\rangle}{T_{2}}-\left\langle\frac{\alpha_{s}}{\pi} G G\right\rangle\left\{\frac{T_{1} T_{2}\left[(1+2 y) T_{1}^{2}+3 T_{2}^{2}+(4 y+2) T_{1} T_{2}\right]}{48\left(T_{1}^{2}+T_{2}^{2}+2 y T_{1} T_{2}\right)^{2}}\right. \\
& \left.-\frac{\left(y^{2}-1\right)\left(T_{1}+T_{2}\right) T_{1}^{3} T_{2}^{2}}{12\left(T_{1}^{2}+T_{2}^{2}+2 y T_{1} T_{2}\right)^{3}}\right\}=K_{\tau}\left(T_{1}, T_{2}, \omega_{c}, \omega_{c}^{\prime} ; y\right),
\end{aligned}
$$

where $T_{1}$ and $T_{2}$ are Borel parameters and

$$
\begin{gathered}
\rho^{\text {pert }}\left(\nu, \nu^{\prime}, y\right)=\frac{3}{2^{7} \pi^{2}}\left(\frac{1}{\sqrt{y^{2}-1}}\right)^{3} \frac{1}{y+1}\left[-3 \nu^{2}+(2 y-1)\left(\nu^{\prime 2}+2 \nu \nu^{\prime}\right)\right] \\
\times \Theta(\nu) \Theta\left(\nu^{\prime}\right) \Theta\left(2 y \nu \nu^{\prime}-\nu^{2}-\nu^{\prime 2}\right) .
\end{gathered}
$$

The integration domain $D$ is constrained by the conditions $\nu \leq \omega_{c}, \nu^{\prime} \leq \omega_{c}^{\prime}$ together with the $\Theta$ functions in (19).

Let us now turn to the derivation of the QCD sum rules for the subleading form factors, $\tau_{1}(y)$ and $\tau_{2}(y)$, defined in (6). To this end, we consider the following three-point correlation functions 


$$
\begin{gathered}
i^{2} \int d^{4} x d^{4} z e^{i\left(k^{\prime} \cdot x-k \cdot z\right)}\left\langle 0\left|T\left(J_{1,+, 3 / 2}^{\nu}(x) \bar{h}_{v^{\prime}}^{(c)} i \overleftarrow{D D} \gamma^{\mu} h_{v}^{(b)}(0) J_{0,-, 1 / 2}^{\dagger}(z)\right)\right| 0\right\rangle=\Xi_{1 V}^{\mu \nu}\left(\omega, \omega^{\prime}, y\right) \\
i^{2} \int d^{4} x d^{4} z e^{i\left(k^{\prime} \cdot x-k \cdot z\right)}\left\langle 0\left|T\left(J_{1,+, 3 / 2}^{\nu}(x) \bar{h}_{v^{\prime}}^{(c)} i \overleftarrow{D} \gamma^{\mu} \gamma_{5} h_{v}^{(b)}(0) J_{0,-, 1 / 2}^{\dagger}(z)\right)\right| 0\right\rangle=\Xi_{1 A}^{\mu \nu}\left(\omega, \omega^{\prime}, y\right) \\
i^{2} \int d^{4} x d^{4} z e^{i\left(k^{\prime} \cdot x-k \cdot z\right)}\left\langle 0\left|T\left(J_{2,+, 3 / 2}^{\alpha \beta}(x) \bar{h}_{v^{\prime}}^{(c)} i \overleftarrow{D D} \gamma^{\mu} h_{v}^{(b)}(0) J_{0,-, 1 / 2}^{\dagger}(z)\right)\right| 0\right\rangle=\Xi_{2 V}^{\mu \alpha \beta}\left(\omega, \omega^{\prime}, y\right) \\
i^{2} \int d^{4} x d^{4} z e^{i\left(k^{\prime} \cdot x-k \cdot z\right)}\left\langle 0\left|T\left(J_{2,+, 3 / 2}^{\alpha \beta}(x) \bar{h}_{v^{\prime}}^{(c)} i \overleftarrow{D} \gamma^{\mu} \gamma_{5} h_{v}^{(b)}(0) J_{0,-, 1 / 2}^{\dagger}(z)\right)\right| 0\right\rangle=\Xi_{2 A}^{\mu \alpha \beta}\left(\omega, \omega^{\prime}, y\right)
\end{gathered}
$$

By saturating the double dispersion integral for the three-point functions in (20) with hadron states and using (10) and (11), one can isolate the contributions from the double pole at $\omega=2 \bar{\Lambda}_{-, 1 / 2}, \omega^{\prime}=2 \bar{\Lambda}_{+, 3 / 2}$ :

$$
\begin{aligned}
\Xi_{1 V}^{\mu \nu}\left(\omega, \omega^{\prime}, y\right) & =\frac{f_{-, \frac{1}{2}} f_{+, 3 / 2}\left[4\left(y \bar{\Lambda}_{+, 3 / 2}-\bar{\Lambda}_{-, 1 / 2}\right) \tau(y) \mathcal{L}_{1 V \tau}^{\mu \nu}+\tau_{1}(y) \mathcal{L}_{1 V \tau_{1}}^{\mu \nu}+\tau_{2}(y) \mathcal{L}_{1 V \tau_{2}}^{\mu \nu}\right]}{\left(2 \bar{\Lambda}_{-, 1 / 2}-\omega-i \epsilon\right)\left(2 \bar{\Lambda}_{+, 3 / 2}-\omega^{\prime}-i \epsilon\right)}+\cdots \\
\Xi_{1 A}^{\mu \nu}\left(\omega, \omega^{\prime}, y\right) & =\frac{f_{-, \frac{1}{2}} f_{+, 3 / 2}\left[4\left(y \bar{\Lambda}_{+, 3 / 2}-\bar{\Lambda}_{-, 1 / 2}\right) \tau(y)-3(y-1)\left(\tau_{1}(y)-\tau_{2}(y)\right)\right] \mathcal{L}_{1 A}^{\mu \nu}}{\left(2 \bar{\Lambda}_{-, 1 / 2}-\omega-i \epsilon\right)\left(2 \bar{\Lambda}_{+, 3 / 2}-\omega^{\prime}-i \epsilon\right)}+\cdots \\
\Xi_{2 V}^{\mu \alpha \beta}\left(\omega, \omega^{\prime}, y\right) & =\frac{f_{-, \frac{1}{2}} f_{+, 3 / 2}\left[\tau_{1}(y)-\tau_{2}(y)\right] \mathcal{L}_{2 V}^{\mu \alpha \beta}}{\left(2 \bar{\Lambda}_{-, 1 / 2}-\omega-i \epsilon\right)\left(2 \bar{\Lambda}_{+, 3 / 2}-\omega^{\prime}-i \epsilon\right)}+\cdots \\
\Xi_{2 A}^{\mu \alpha \beta}\left(\omega, \omega^{\prime}, y\right) & =\frac{f_{-, \frac{1}{2}} f_{+, 3 / 2}\left[\tau_{1}(y) \mathcal{L}_{2 A \tau_{1}}^{\mu \alpha \beta}+\tau_{2}(y) \mathcal{L}_{2 A \tau_{2}}^{\mu \alpha \beta}\right]}{\left(2 \bar{\Lambda}_{-, 1 / 2}-\omega-i \epsilon\right)\left(2 \bar{\Lambda}_{+, 3 / 2}-\omega^{\prime}-i \epsilon\right)}+\cdots
\end{aligned}
$$

where the Lorentz structure $\mathcal{L}^{\mu \nu}$ 's and $\mathcal{L}^{\mu \alpha \beta}$ 's are collected in the Appendix.

On the other hand,it turns out that the calculation of these correlators with the operator product expansion (OPE) in HQET yields the following general structure

$$
\begin{aligned}
\Xi_{1 V}^{\mu \nu}\left(\omega, \omega^{\prime}, y\right) & =\Xi_{\tilde{\tau}} \mathcal{L}_{1 V \tau}^{\mu \nu}+\Xi_{\tau_{1}} \mathcal{L}_{1 V \tau_{1}}^{\mu \nu}+\Xi_{\tau_{2}} \mathcal{L}_{1 V \tau_{2}}^{\mu \nu} \\
\Xi_{1 A}^{\mu \nu}\left(\omega, \omega^{\prime}, y\right) & =\left[\Xi_{\tilde{\tau}}-3(y-1)\left(\Xi_{\tau_{1}}-\Xi_{\tau_{2}}\right)\right] \mathcal{L}_{1 A}^{\mu \nu}, \\
\Xi_{2 V}^{\mu \alpha \beta}\left(\omega, \omega^{\prime}, y\right) & =\left(\Xi_{\tau_{1}}-\Xi_{\tau_{2}}\right) \mathcal{L}_{2 V}^{\mu \alpha \beta} \\
\Xi_{2 A}^{\mu \alpha \beta}\left(\omega, \omega^{\prime}, y\right) & =\Xi_{\tau_{1}} \mathcal{L}_{2 A \tau_{1}}^{\mu \alpha \beta}+\Xi_{\tau_{2}} \mathcal{L}_{2 A \tau_{2}}^{\mu \alpha \beta}
\end{aligned}
$$

where the coefficient functions $\Xi_{\tau_{1}}, \Xi_{\tau_{2}}$ and $\Xi_{\tilde{\tau}}$ are scalar analytic functions in the off-shell energies $\omega$ and $\omega^{\prime}$.

Comparing (21) with (22) one can see that they are compatible. Therefore, we can calculate the scalar functions $\Xi_{i}\left(\omega, \omega^{\prime}, y\right)$ with the QCD sum rules in HQET. From (22) and (21) one can see that the sum rules for $\Xi_{\tau_{1}}, \Xi_{\tau_{2}}$ and $\Xi_{\tilde{\tau}}$ yield sum rules for $\tau_{1}(y), \tau_{2}(y)$ and $\left(y \bar{\Lambda}_{+, 3 / 2}-\bar{\Lambda}_{-, 1 / 2}\right) \tau(y)$, respectively. In the theoretical calculation, for simplicity, the residual momentum $k$ is chosen to be parallel to $v$ such that $k_{\mu}=(k \cdot v) v_{\mu}$ (and similar for $k^{\prime}$ ). The theoretical expression for the correlator in HQET consists of a perturbative part and contributions from vacuum condensations. Confining us to the leading order of 
perturbation and the operators with dimension $D \leq 5$ in OPE, the relevant Feynman diagrams are shown in Fig 1 . We shall focus, at first, on the coefficient functions $\Xi_{\tau_{1}}\left(\omega, \omega^{\prime}, y\right)$ and $\Xi_{\tau_{2}}\left(\omega, \omega^{\prime}, y\right)$ to construct the sum rules for the subleading form factors $\tau_{1}(y)$ and $\tau_{2}(y)$.

The spectral densities in the double dispersion integral for the perturbative diagram depicted in Fig. 1(a) turn out to be

$$
\begin{aligned}
\rho_{\tau_{1}}^{\text {pert }}\left(\nu, \nu^{\prime}, y\right)= & \frac{3}{2^{8} \pi^{2}}\left(\frac{1}{\sqrt{y^{2}-1}}\right)^{5} \frac{1}{y+1}\left[-5 \nu^{3}+(12 y-3) \nu^{2} \nu^{\prime}-\left(6 y^{2}-6 y+3\right) \nu \nu^{\prime 2}\right. \\
& \left.-\left(2 y^{2}-2 y+1\right) \nu^{\prime 3}\right] \Theta(\nu) \Theta\left(\nu^{\prime}\right) \Theta\left(2 y \nu \nu^{\prime}-\nu^{2}-\nu^{\prime 2}\right) \\
\rho_{\tau_{2}}^{\text {pert }}\left(\nu, \nu^{\prime}, y\right)= & \frac{3}{2^{8} \pi^{2}}\left(\frac{1}{\sqrt{y^{2}-1}}\right)^{5} \frac{1}{y+1}\left[(4 y-1) \nu^{3}-\left(9 y^{2}-6 y\right) \nu^{2} \nu^{\prime}+\left(4 y^{3}-8 y^{2}\right.\right. \\
& \left.+2 y-1) \nu \nu^{\prime 2}+\left(2 y^{3}-y^{2}+2 y\right) \nu^{\prime 3}\right] \Theta(\nu) \Theta\left(\nu^{\prime}\right) \Theta\left(2 y \nu \nu^{\prime}-\nu^{2}-\nu^{\prime 2}\right)
\end{aligned}
$$

The non-perturbative power corrections to the correlators are computed from the diagrams involving the quark and gluon condensates in Fig. 1(b)-(c) in the Fock-Schwinger gauge $x_{\mu} A^{\mu}(x)=0$. We find that the only non-vanishing contribution is the gluon condensate. After adding the non-perturbative part and making the double Borel transformations one obtains the sum rules for $\tau_{1}(y)$ and $\tau_{2}(y)$ as follows

$$
\begin{aligned}
\tau_{1}(y) f_{-, 1 / 2} f_{+, 3 / 2} e^{-2\left(\bar{\Lambda}_{-, 1 / 2} / T_{1}+\bar{\Lambda}_{+, 3 / 2} / T_{2}\right)}= & \int_{D} d \nu d \nu^{\prime} \rho_{\tau_{1}}^{\text {pert }}\left(\nu, \nu^{\prime}, y\right) e^{-\nu / T_{1}-\nu^{\prime} / T_{2}} \\
& -\left\langle\frac{\alpha_{s}}{\pi} G G\right\rangle H_{\tau_{1}}\left(T_{1}, T_{2}\right), \\
\tau_{2}(y) f_{-, 1 / 2} f_{+, 3 / 2} e^{-2\left(\bar{\Lambda}_{-, 1 / 2} / T_{1}+\bar{\Lambda}_{+, 3 / 2} / T_{2}\right)}= & \int_{D} d \nu d \nu^{\prime} \rho_{\tau_{2}}^{\text {pert }}\left(\nu, \nu^{\prime}, y\right) e^{-\nu / T_{1}-\nu^{\prime} / T_{2}} \\
& -\left\langle\frac{\alpha_{s}}{\pi} G G\right\rangle H_{\tau_{2}}\left(T_{1}, T_{2}\right),
\end{aligned}
$$

where

$$
\begin{aligned}
H_{\tau_{1}}\left(T_{1}, T_{2}\right)= & \frac{1}{48} T_{1}^{3} T_{2}\left[-T_{1}^{5}+4 y T_{1}^{4} T_{2}+(8 y+14) T_{1}^{3} T_{2}^{2}+\left(4 y^{2}+12 y+16\right) T_{1}^{2} T_{2}^{3}\right. \\
& \left.+(16 y+3) T_{1} T_{2}^{4}+4 T_{2}^{5}\right] /\left(T_{1}^{2}+2 y T_{1} T_{2}+T_{2}^{2}\right)^{4}, \\
H_{\tau_{2}}\left(T_{1}, T_{2}\right)= & \frac{1}{96} T_{1}^{2} T_{2}\left[(2 y+1) T_{1}^{6}-\left(8 y^{2}-8 y\right) T_{1}^{5} T_{2}-(28 y-9) T_{1}^{4} T_{2}^{2}+-24 y^{2} T_{1}^{3} T_{2}^{3}\right. \\
& \left.-\left(8 y^{2}+6 y-11\right) T_{1}^{2} T_{2}^{4}+8 y T_{1} T_{2}^{5}+3 T_{2}^{6}\right] /\left(T_{1}^{2}+2 y T_{1} T_{2}+T_{2}^{2}\right)^{4}
\end{aligned}
$$

The integration domain $D$ is restricted to the area in $\nu \leq \omega_{c}, \nu^{\prime} \leq \omega_{c}^{\prime}$.

We have checked that the sum rules for $\tau_{1}(y)$ and $\tau_{2}(y)$ derived from Eqs. (22a and (22d) are the same and they are also consistant with the sum rules derived for $\tau_{1}-\tau_{2}$ from Eqs. (22b) and (22c).

Furthermore, from the coefficient function $\Xi_{\tilde{\tau}}$ in $(22 \mathrm{a})$ and $(22 \mathrm{~b})$ one finds the same sum rule for the combination $\left(y \bar{\Lambda}_{+, 3 / 2}-\bar{\Lambda}_{-, 1 / 2}\right) \tau(y)$ as follows 


$$
\begin{aligned}
& \left(y \bar{\Lambda}_{+, 3 / 2}-\bar{\Lambda}_{-, 1 / 2}\right) \tau(y) f_{-, 1 / 2} f_{+, 3 / 2} e^{-2\left(\bar{\Lambda}_{-, 1 / 2} / T_{1}+\bar{\Lambda}_{+, 3 / 2} / T_{2}\right)}= \\
& -\frac{1}{2}\left(y \frac{\partial}{\partial T_{2}^{-1}}-\frac{\partial}{\partial T_{1}^{-1}}\right) K_{\tau}\left(T_{1}, T_{2}, \omega_{c}, \omega_{c}^{\prime} ; y\right)
\end{aligned}
$$

where $K_{\tau}$ in this equation is identical to the function $K_{\tau}$ in (18). Therefore, (29) is consistant with the sum rule (18) in the leading order,

The above consistancy checks confirm that the correlators have the forms in (22) and that our method is consistant with the general analysis of Ref. [4] described in Sec. II].

In obtaining the sum rules (25), (26) and (29) the quark-hadron duality has been assumed. In doing this, the contribution from higher hadronic states is simulated by the perturbative part above some threshold energy. In the QCD sum rule analysis for IsgurWise functions for $B$ semileptonic decays into ground state $D$ mesons, it is argued by the authors of [2,6, [7] that the perturbative and the hadronic spectral densities can not be locally dual to each other, the necessary way to restore duality is to integrate the spectral densities over the "off-diagonal" variable $\nu_{-}=\left(\nu-\nu^{\prime}\right) / 2$, keeping the "diagonal" variable $\nu_{+}=\left(\nu+\nu^{\prime}\right) / 2$ fixed. It is in $\nu_{+}$that the quark-hadron duality is assumed for the integrated spectral densities. In doing this, for simplicity, the two Borel parameters are taken to be equal: $T_{1}=T_{2}=2 T$. We shall use the same prescription here.

The $\Theta$ functions in (23) and (24) imply that in terms of $\nu_{+}$and $\nu_{-}$the double discontinuities of the corrrelator are confined to the region $-\sqrt{y^{2}-1} /(1+y) \nu_{+} \leq \nu_{-} \leq$ $\sqrt{y^{2}-1} /(1+y) \nu_{+}$and $\nu_{+} \geq 0$. According to our prescription an isosceles triangle with the base $\nu_{+}=\nu_{c}$ is retained in the integation domain of the perturbative term in the sum rule.

In view of the asymmetry of the problem at hand with respect to the initial and final states one may attempt to use an asymmetric triangle in the perturbative integral. However, in that case the factor $\left(y^{2}-1\right)^{3 / 2}$ in the denominator of (23) and (24) is not canceled after the integration so that the Isgur-Wise function or it's derivative will be divergent at $y=1$. Similar situation occurs for the sum rule of the Isgur-Wise functions for the tansition between ground states if a different domain is taken in the perturbative integal [7.

Putting everything together one obtains the final expressions for the QCD sum rules

$$
\begin{aligned}
& \tau_{1}(y) f_{-, \frac{1}{2}} f_{+, \frac{3}{2}} e^{-\left(\bar{\Lambda}_{-, \frac{1}{2}}+\bar{\Lambda}_{+, \frac{3}{2}}\right) / T}=- \frac{3}{8 \pi^{2}} \frac{1}{(y+1)^{4}} \int_{0}^{\omega_{c}} d \omega_{+} \omega_{+}^{4} e^{-\omega_{+} / T} \\
&+\frac{1}{3 \times 2^{5}}\left\langle\frac{\alpha_{s}}{\pi} G G\right\rangle \frac{y+9}{(y+1)^{3}} T \\
& \tau_{2}(y) f_{-, \frac{1}{2}} f_{+, \frac{3}{2}} e^{-\left(\bar{\Lambda}_{-, \frac{1}{2}}+\bar{\Lambda}_{+, \frac{3}{2}}\right) / T}=\frac{1}{16 \pi^{2}} \frac{5 y-1}{(y+1)^{4}} \int_{0}^{\omega_{c}} d \omega_{+} \omega_{+}^{4} e^{-\omega_{+} / T} \\
&-\frac{1}{3 \times 2^{5}}\left\langle\frac{\alpha_{s}}{\pi} G G\right\rangle \frac{5 y-3}{(y+1)^{3}} T .
\end{aligned}
$$


We end this section by noting that the QCD $O\left(\alpha_{s}\right)$ corrections have not been included in the sum rule calculations. However, the Isgur-Wise function obtained from the QCD sum rule actually is the ratio of the three-point correlator to the two-point correlator. While both of these correlators subject to large perturbative QCD corrections, it is expected that their ratio is not much affected by these corrections because of cancelation. This has been proved to be true in the analysis for $B$ semileptonic decay to ground state heavy mesons 【7.

\section{NUMERICAL ANALYSIS AND CONCLUSION}

In order to obtain the values for $\tau_{1}(y)$ and $\tau_{2}(y)$ from Eqs. (30) and (31) in the numerical evaluation we need to use the hadronic parameters $\bar{\Lambda}$ 's and $f$ 's of the corresponding interpolating currents as input. The QCD sum rules derived from the two-point correlator has been applied to determine $\bar{\Lambda}$ and $f . \bar{\Lambda}_{-, 1 / 2}$ and $f_{-, 1 / 2}$ can be obtained from the results in [7] as $\bar{\Lambda}_{-, 1 / 2}=0.5 \mathrm{GeV}$ and $f_{-, 1 / 2} \simeq 0.24 \mathrm{GeV}^{3 / 2}$ at the order $\alpha_{s}=0$. Notice that the coupling constant $f_{-, 1 / 2}$ defined in the present work is a factor $1 / \sqrt{2}$ smaller than that defined in [7]. Determination of $\bar{\Lambda}_{+, 3 / 2}$ and $f_{+, 3 / 2}$ by QCD sum rules gave the results: $\bar{\Lambda}_{+, 3 / 2}=0.95 \mathrm{GeV}$ and $f_{+, 3 / 2}=0.19 \mathrm{GeV}^{5 / 2}$ at the order $\alpha_{s}=0$ [20,21]. For the QCD parameters entering the theoretical expressions, we take the standard values: $\left\langle\alpha_{s} G G\right\rangle=(0.04) \mathrm{GeV}^{4}$.

Imposing usual criterium that both higher-order power corrections and the contribution of the continuum should not be very large, we find an acceptable stability window for the threshold parameter in the range $\omega_{c}=2.0-2.6 \mathrm{GeV}$, in which the results do not appreciably depend on the Borel parameter in the range $T=0.7-1.1 \mathrm{GeV}$. The range of Borel parameter here overlaps with that of the corresponding two-point sum rules [7,20].

The values of the form factors $\tau_{1}(y)$ and $\tau_{2}(y)$ at zeor recoil as functions of the Borel parameter are shown in Fig. 2(a) and 3(a), for three different values of the continuum threshold $\omega_{c}$. The numerical results for $\tau_{1}(y)$ and $\tau_{2}(y)$ are shown in Fig. 2(b) and 3(b), where the curves refer to three different values of $\omega_{c}$ and $T$ is fixed at $T=0.9 \mathrm{GeV}$.

The numerical analysis shows that $\tau_{1}(y)$ and $\tau_{2}(y)$ are slowly varying functions in the allowed kinematic range for $B \rightarrow D_{1} \ell \bar{\nu}$ and $B \rightarrow D_{2}^{*} \ell \bar{\nu}$ decays. The resulting curves for $\tau_{1}(y)$ and $\tau_{2}(y)$ may be well parameterized by the linear approximations

$$
\begin{aligned}
& \tau_{1}(y)=\tau_{1}(1)\left(1-\rho_{\tau_{1}}^{2}(y-1)\right), \quad \tau_{1}(1)=-0.4 \pm 0.1, \quad \rho_{\tau_{1}}^{2}=1.4 \pm 0.2, \\
& \tau_{2}(y)=\tau_{2}(1)\left(1-\rho_{\tau_{2}}^{2}(y-1)\right), \quad \tau_{2}(1)=0.28 \pm 0.05, \quad \rho_{\tau_{2}}^{2}=0.5 \pm 0.1 \text {. }
\end{aligned}
$$

The errors here reflect only the uncertainty due to $\omega_{c}$ and $T$. They do not contain other errors in the QCD sum rule approach. 
In conclusion, we have presented a QCD sum rule analysis of the subleading Isgur-Wise functions $\tau_{1}(y)$ and $\tau_{2}(y)$, appearing in the heavy quark expansion of the transition matrix elements between heavy mesons due to matching of the weak currents in QCD onto those in the effective theory at the order $1 / m_{Q}$. Our approach is in accordance with the general relations obtained from analysis based on HQET in [4].

\section{ACKNOWLEDGMENTS}

This work was supported in part by the National Natural Science Foundation of China. 


\section{APPENDIX:}

We list here the lorentz structures used in the paper.

$$
\begin{aligned}
\mathcal{L}_{1 V \tau}^{\mu \nu} & =\frac{1}{\sqrt{6}}\left[-(y+1) g_{t}^{\mu \nu}+v^{\prime \mu} v_{t}^{\nu}\right] \\
\mathcal{L}_{1 V \tau_{1}}^{\mu \nu} & =\frac{1}{\sqrt{6}}\left\{3\left(y^{2}-1\right) g_{t}^{\mu \nu}-\left[(4 y-1) v^{\mu}-(y+2) v^{\prime \mu}\right] v_{t}^{\nu}\right\} \\
\mathcal{L}_{1 V \tau_{2}}^{\mu \nu} & =\frac{1}{\sqrt{6}}\left\{-3\left(y^{2}-1\right) g_{t}^{\mu \nu}-\left[5 v^{\mu}-(3 y+2) v^{\prime \nu}\right] v_{t}^{\nu}\right\} \\
\mathcal{L}_{1 A}^{\mu \nu} & =\frac{-i}{\sqrt{6}} \varepsilon^{\mu \nu \alpha \beta} v_{\alpha} v_{\beta}^{\prime} \\
\mathcal{L}_{2 V}^{\mu \alpha \beta} & =\frac{i}{2}\left(\varepsilon^{\mu \alpha \rho \sigma} v_{\beta}^{t}+\varepsilon^{\mu \beta \rho \sigma} v_{\alpha}^{t}\right) v_{\rho} v_{\sigma}^{\prime}, \\
\mathcal{L}_{2 A \tau_{1}}^{\mu \alpha \beta} & =(y-1)\left\{\frac{1}{2}\left(g_{t}^{\alpha \mu} v_{t}^{\beta}+g_{t}^{\beta \mu} v_{t}^{\alpha}\right)-\frac{1}{3} g_{t}^{\alpha \beta} v_{t}^{\mu}\right\}+\left(2 v^{\mu}+v^{\prime \mu}\right)\left\{v_{t}^{\alpha} v_{t}^{\beta}-\frac{1}{3}\left(1-y^{2}\right) g_{t}^{\alpha \beta}\right\}, \\
\mathcal{L}_{2 A \tau_{2}}^{\mu \alpha \beta} & =-(y-1)\left\{\frac{1}{2}\left(g_{t}^{\alpha \mu} v_{t}^{\beta}+g_{t}^{\beta \mu} v_{t}^{\alpha}\right)-\frac{1}{3} g_{t}^{\alpha \beta} v_{t}^{\mu}\right\}+v^{\prime \mu}\left\{v_{t}^{\alpha} v_{t}^{\beta}-\frac{1}{3}\left(1-y^{2}\right) g_{t}^{\alpha \beta}\right\},
\end{aligned}
$$

where $g_{t}^{\alpha \beta}=g^{\alpha \beta}-v^{\prime \alpha} v^{\prime \beta}$ and $v_{t}^{\alpha}=v^{\alpha}-y v^{\prime \alpha}$. It is easy to see that these Lorentz structures satisfy

$$
\begin{array}{r}
v_{\nu}^{\prime} \mathcal{L}_{1 V \tau}^{\mu \nu}=v_{\nu}^{\prime} \mathcal{L}_{1 V \tau_{1}}^{\mu \nu}=v_{\nu}^{\prime} \mathcal{L}_{1 V \tau_{2}}^{\mu \nu}=v_{\nu}^{\prime} \mathcal{L}_{1 A}^{\mu \nu}=0 \\
g_{\alpha \beta} \mathcal{L}_{2 V}^{\mu \alpha \beta}=g_{\alpha \beta} \mathcal{L}_{2 A \tau_{1}}^{\mu \alpha \beta}=g_{\alpha \beta} \mathcal{L}_{2 A \tau_{2}}^{\mu \alpha \beta}=0 \\
v_{\alpha}^{\prime} \mathcal{L}_{2 V}^{\mu \alpha \beta}=v_{\alpha}^{\prime} \mathcal{L}_{2 A \tau_{1}}^{\mu \alpha \beta}=v_{\alpha}^{\prime} \mathcal{L}_{2 A \tau_{2}}^{\mu \alpha \beta}=0 \\
v_{\beta}^{\prime} \mathcal{L}_{2 V}^{\mu \alpha \beta}=v_{\beta}^{\prime} \mathcal{L}_{2 A \tau_{1}}^{\mu \alpha \beta}=v_{\beta}^{\prime} \mathcal{L}_{2 A \tau_{2}}^{\mu \alpha \beta}=0
\end{array}
$$

The appearence of the Lorentz structures satisfying these relations is the result of the following equations

$$
\begin{aligned}
& v_{\nu}^{\prime} J_{1,+, 3 / 2}^{\nu}=v_{\alpha}^{\prime} J_{2,+, 3 / 2}^{\alpha \beta}=0 \\
& g_{\alpha \beta} J_{2,+, 3 / 2}^{\alpha \beta}=0, \quad J_{2,+, 3 / 2}^{\alpha \beta}=J_{2,+, 3 / 2}^{\beta \alpha},
\end{aligned}
$$

satisfied by the interpolating currents in the correlators. 


\section{REFERENCES}

[1] B. Grinstein, Nucl. Phys. B339, 253 (1990); E. Eichten and B. Hill, Phys. Lett. B234, 511 (1990); A. F. Falk, H. Georgi, B. Grinstein and M. B. Wise, Nucl. Phys. B343, 1 (1990); F. Hussain, J. G. Körner, K. Schilcher, G. Thompson and Y. L. Wu, Phys. Lett. B249, 295 (1990); J. G. Körner and G. Thompson, Phys. Lett. B264, 185 (1991).

[2] For a review, see: M. Neubert, Phys. Rep. 245, 259 (1994).

[3] N. Isgur and M.B. Wise, Phys. Rev. D43, 819 (1991).

[4] A.K. Leibovich, Z. Ligeti, I.W. Steward and M.B. Wise, Phys. Rev. Lett. 78, 3995 (1997); A.K. Leibovich, Z. Ligeti, I.W. Steward and M.B. Wise, Phys. Rev. D57, 308 (1998).

[5] E. Bagan, P. Ball, V. M. Braun and H. G. Dosch, Phys. Lett. B278, 457 (1992); D. J. Broadhurst and A. G. Grozin, Phys. Lett. B274, 421 (1992); E. Bagan, P. Ball and P. Gosdzinsky, Phys. Lett. B301 249 (1993).

[6] B. Blok and M. Shifman, Phys. Rev. D47 2949 (1993).

[7] M. Neubert, Phys. Rev. D 45, 2451 (1992); M. Neubert, Phys. Rev. D 46, 3914 (1992); M. Neubert, Phys. Rev. D 47, 4063 (1993); Z. Ligeti, Y. Nir and M. Neubert, Phys. Rev. D 49, 4063 (1994).

[8] P. Colangelo, G. Nardulli, A. A. Ovchinnikov and N. Paver, Phys. Lett. B269, 201 (1991); P. Colangelo, G. Nardulli and N. Paver, Phys. Lett. B293, 207 (1992).

[9] M. Q. Huang and Y. B. Dai Phys. Rev. D 59, 034018 (1999).

[10] P. Colangelo, F. De Fazio and N. Paver, Phys. Rev. D 58, 116005 (1998).

[11] S. Godfrey and N. Isgur, Phys. Rev. D32, 189 (1985).

[12] N. Isgur, D. Scora, B. Grinstein and M.B. Wise D39, 799 (1989).

[13] P. Cea, P. Colangelo, L. Cosmai and G. Nardulli, Phys. Lett. B 206, 691 (1988); P. Colangelo, G. Nardulli and M. Pietroni, Phys. Rev. D43, 3002 (1991).

[14] A. Wambach, Nucl. Phys. B434 647 (1995).

[15] S. Veseli and I. Dunietz, Phys. Rev. D54, 6803 (1996); S. Veseli and M. G. Olsson, Phys. Rev. D54, 886 (1996).

[16] V. Morenas, A. Le Yaouanc, L. Oliver, O. Pène and J.-C. Raynal, Phys. Rev. D56, 5668 (1997). 
[17] A. Deandrea, N. Di Bartolomeo, R. Gatto, G. Nardulli and A. D. Polosa, Phys. Rev. D 58, 034004 (1998).

[18] Y. B. Dai, C. S. Huang and H. Y. Jin, Zeit. Phys. C60, 527 (1993).

[19] A. F. Falk, Nucl. Phys. B378, 79(1992).

[20] Y. B. Dai, C. S. Huang, M. Q. Huang and C. Liu, Phys. Lett. B390, 350 (1997); Y. B. Dai, C. S. Huang and M. Q. Huang, Phys. Rev. D 55, 5719 (1997);

[21] Y. B. Dai, C. S. Huang, M. Q. Huang, H. Y. Jin and C. Liu, Phys. Rev. D 58, 094032 (1998). 


\section{Figure Captions}

Fig. 1. Feynman diagrams contributing to the sum rules for the IsgurWise form factors in the coordinate gauge. The gray square corresponds to the insertion of the $1 / m_{c}$ current.

Fig. 2. Numerical evaluation for the sum rule (30): (a) dependence of $\tau_{1}(1)$ on the Borel parameter $T$ for defferent values of the continuum threshold $\omega_{c}$; (b) Results for the Isgur-Wise form factor $\tau_{1}(y)$ with $T=$ $0.9 \mathrm{GeV}$.

Fig. 3. Numerical evaluation for the sum rule (31): (a) dependence of $\tau_{2}(1)$ on the Borel parameter $T$ for defferent values of the continuum threshold $\omega_{c}$; (b) Results for the Isgur-Wise form factor $\tau_{2}(y)$ with $T=$ $0.9 \mathrm{GeV}$. 
FIGURES

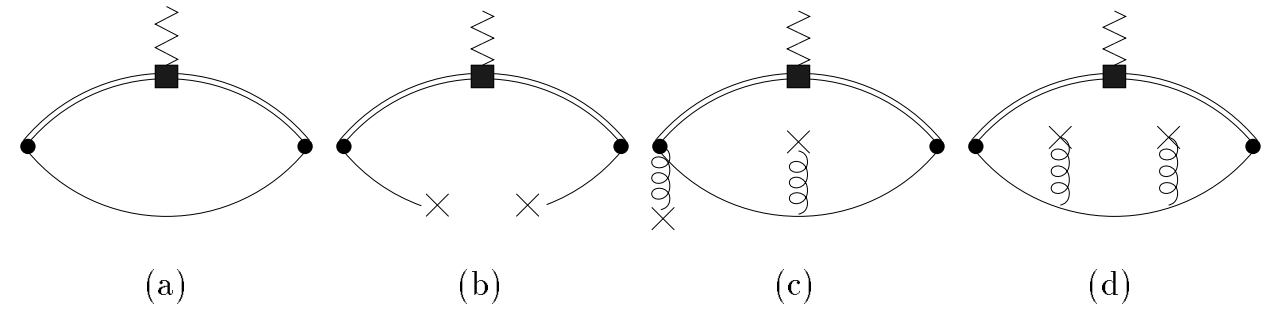

Fig 1 

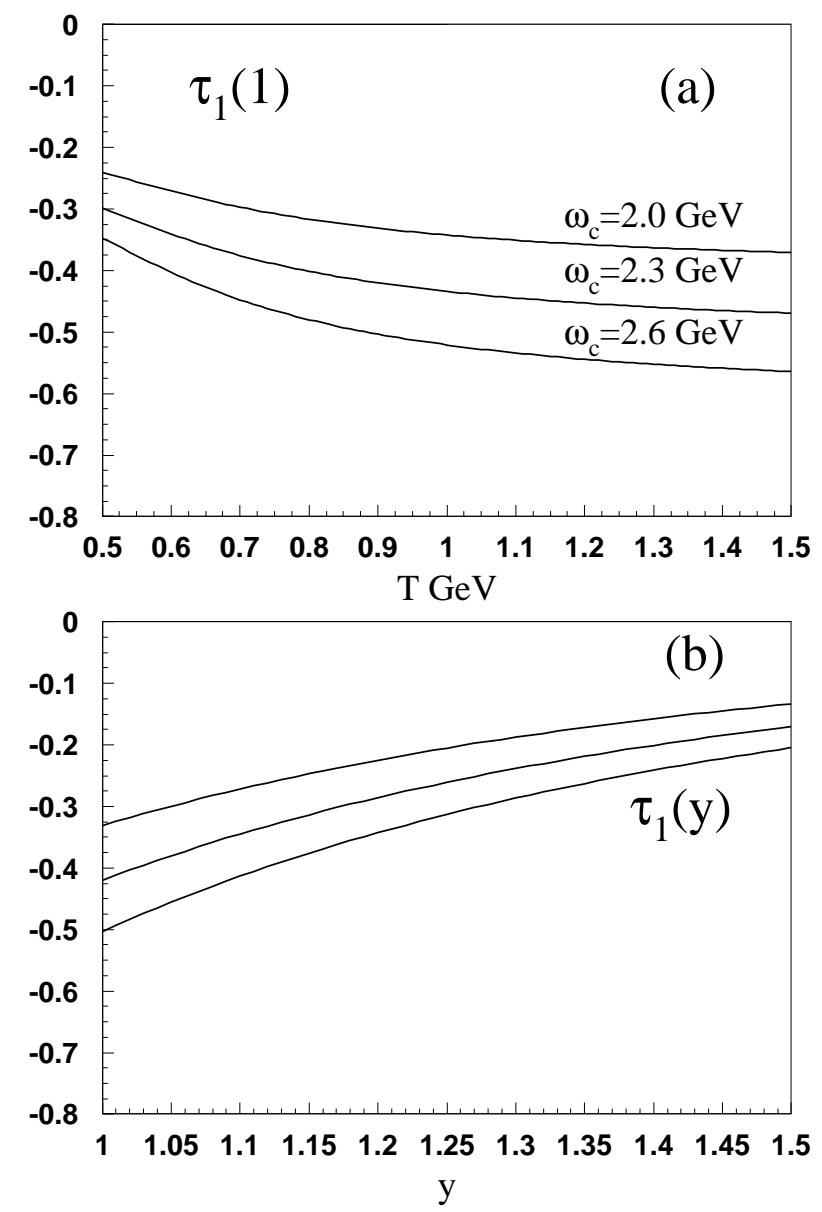

Fig. 2 

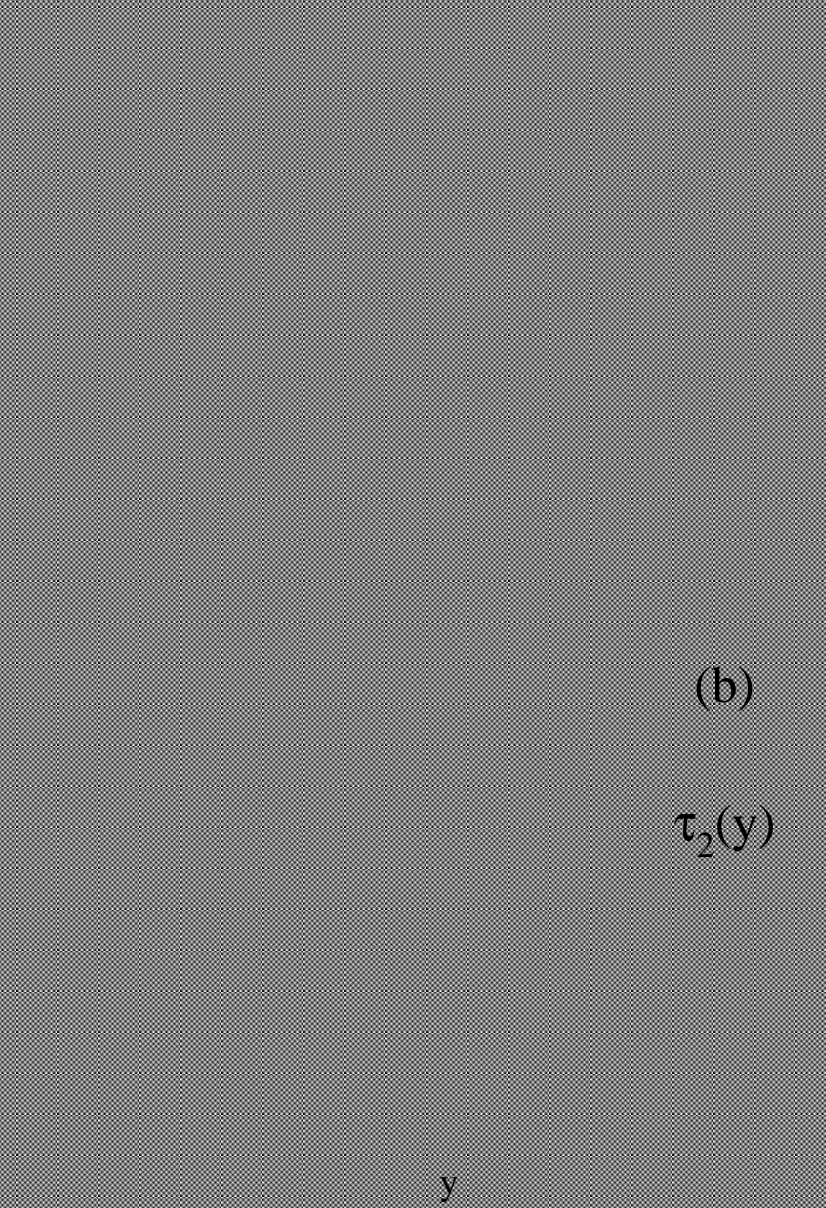

Fig. 3 